\title{
Estimation of a Matrix of Heterogeneity Parameters in Multivariate Meta-Analysis of Random-Effects Models *
}

\author{
Abera Wouhib \\ National Center for Health Statistics, Centers for Disease Control and Prevention, \\ 3311 Toledo Road, Hyattsville, MD, 20782 \\ awouhib@cdc.gov
}

Received 7 July 2013

Accepted 7 November 2013

\begin{abstract}
Multivariate meta-analysis has potential over its univariate counterpart. The most common challenge in univariate or multivariate meta-analysis is estimating heterogeneity parameters in non-negative domains under the random-effects model assumption. In this context, two new multivariate estimation methods are demonstrated; first, by extending the Sidik and Jonkman (2005) univariate estimates to a multivariate setting, and second, by considering an iterative version of the Sidik and Jonkman method, namely, a Hybrid method developed in Wouhib (2013). These two methods are compared with extended DerSimonian and Laird methods (Jackson et al. 2009; Chen et al. 2012) by using an example and simulation in random-effects multivariate meta-analysis. Finally, the benefits of the proposed estimates are evaluated in terms of precision in estimating vectors of effect sizes and associated covariance matrices via simulation. Also, some limitations and remedies resulting from negative definite matrix in estimating heterogeneity parameters will be discussed.
\end{abstract}

Keywords: DerSimonian and Laird; Heterogeneity Parameter; Hybrid Method; Random Effects; Sidik and Jonkman.

2000 Mathematics Subject Classification: 22E46, 53C35, 57S20

\section{Introduction}

Multivariate meta-analysis has an abundance of potential and promise over the univariate metaanalysis including one-way and meta-regression models. In particular, it can describe the association between the estimates of effects in order to make predictions about the true effects of a new study and provide estimates with better statistical properties than univariate due to the borrowing of strength that it enables (Jackson et al. 2011). There are two scenarios that would warrant consideration of a multivariate approach in meta-analysis. First, technically equivalent outcomes may not be considered as a single entity because of differences in study design, aims or definitions. Second, because outcomes may be strongly related among themselves, an evaluation of the agent should 'borrow strength' not only across studies, as in traditional meta-analysis models, but also across outcomes. The rationale to synthesizing coefficients of several independent regressions could be a multivariate meta-analysis problem. Becker and $\mathrm{Wu}$ (2007) stated in their paper that the synthesis of regression slopes has received increased attention in recent years, and can be handled in multivariate meta-analysis as long as the regression variables are measured in a similar way across independent studies.

*Disclaimer: The findings and conclusions stated in this manuscript are solely those of the author. They do not necessarily reflect the views of the National Center for Health Statistics or the Centers for Disease Control and Prevention. 


\section{A. Wouhib}

This paper begins with a discussion of the multivariate random-effects model and its practical merit that has become more prevalent in meta-analyses in recent years. The validity of the estimators and their adequacy or inadequacy is assessed by discussing the most recent methods of estimating a matrix of heterogeneity parameters in a random-effects setting, which includes the multivariate version of the DerSimonian and Laird (DL) method proposed by Jackson et al. (2009) and Chen et al. (2012). Also two new methods are proposed, namely, a multivariate extension of the Sidik and Jonkman (SJ) estimator and its iterative version called the Hybrid estimator (Wouhib, 2013). In addition to the theoretical justification, the general approach in measuring the effectiveness of a new method is examined by comparing it to the estimated mean effect sizes and associated covariance matrices offered by other methods using simulation. Details of the SJ method along with the hybrid method, will be discussed by presenting a generalized least squares (GLS) approach with remarks on the challenges and limitations in synthesizing effect sizes in multivariate modeling.

The paper is organized as follows. Section 2 outlines the estimation of a matrix of heterogeneity parameters based on extending the DL method for a random-effects multivariate meta-analysis. Details of the two methods presented by Jackson et al. (2009) and Chen et al. (2012), with their respective issues and limitations, are discussed here. The newly proposed methods, namely, the multivariate extension of the SJ method and the Hybrid method, are also introduced and explained in this section. Section 3 emphasizes an application by using a two dimensional example taken from a recent paper of Jackson et al. (2013) and another three dimensional example from Chen et al. (2012). In Section 4, simulation results are presented by using the estimates from the three dimensional example as true population parameters. Finally, further discussion and issues that can arise from estimating the matrix of the heterogeneity parameters are given in Section 5 by highlighting the major findings of the research as a conclusion.

\section{Methods}

A random-effects meta-analysis model can be visualized in a p-dimensional multivariate setting, by simply extending the one-way univariate meta-analysis model. Let the $i^{\text {th }}$ variable $\mathrm{b}_{\mathrm{i}}$ be a $\mathrm{p}$ dimensional correlated vector of study $i=1,2, \ldots k$. The vector $\mathrm{b}_{\mathrm{i}}$ is assumed to be distributed $\mathscr{N}\left(\beta_{i}, \Sigma_{i}\right)$. Also, let the p-dimensional population vector $\beta_{i}$ be assumed to be random and distributed $\beta_{i} \sim \mathscr{N}(\beta, \mathrm{T})$, where $\Sigma_{i}$ and $\mathrm{T}$ are the $p \times p$ matrices of the within-study and between-study covariances, respectively, and the $\mathrm{p}$-dimensional vector, $\beta$, is the overall unknown true effect size to be estimated. Then, the random-effects model

$$
\mathrm{b}_{\mathrm{i}}=\beta+v_{\mathrm{i}}+\varepsilon_{\mathrm{i}}
$$

is a combination of the two models where

$$
v_{\mathbf{i}} \sim \mathscr{N}(0, \mathbf{T}), \varepsilon_{\mathbf{i}} \sim \mathscr{N}\left(0, \Sigma_{\mathbf{i}}\right)
$$

and

$$
v_{\mathbf{i}} \perp \varepsilon_{\mathbf{i}}
$$

for all $i=1,2, \ldots k$. 
The random-effects multivariate model can be generalized as

$$
\mathbf{b}=\mathrm{Z} \beta+\mathrm{u}+\mathrm{v},
$$

where the vectors $\mathbf{b}, \beta$, and $\mathbf{u}+\mathbf{v}$ along with a matrix $\mathbf{Z}$ are defined to be:

$$
\begin{gathered}
\mathbf{b}=\left(b_{11} \ldots b_{1 p} \ldots b_{k 1} \ldots b_{k p}\right)_{k p \times 1}^{\prime}, \\
\beta=\left(\beta_{1} \ldots \beta_{p}\right)_{\mathbf{p} \times \mathbf{1}}^{\prime}, \\
\mathbf{u}+\mathbf{v}=\left(u_{11}+v_{1} \ldots u_{1 p}+v_{p} \ldots u_{k p}+v_{p}\right)_{k p \times 1}^{\prime}
\end{gathered}
$$

and

$$
\mathbf{Z}=\left(I_{p \times p} \ldots I_{p \times p} \ldots I_{p \times p}\right)_{k p \times p}^{\prime} .
$$

From the model, the covariance matrix of the additive error terms, $(\mathrm{u}+\mathrm{v})$, is a $k p \times k p$ dimensional diagonal box matrix,

$$
\Delta=\operatorname{Diag}\left(\Delta_{1} \cdots \Delta_{i} \cdots \Delta_{k}\right)_{k p \times k p}
$$

and

$$
\Delta_{i}=\Sigma_{i}+T
$$

with $\Delta_{i}$ being a $p \times p$ matrix for study $i=1,2, \ldots, k$. is

Once the models are set, the generalized least square estimate of a $p \times 1$ dimensional vector, $\beta$,

$$
\hat{\beta}=\left(Z^{\prime} \Delta^{-1} Z\right)^{-1}\left(Z^{\prime} \Delta^{-1} b\right) .
$$

In parallel with earlier developments of the methods of meta-analysis, when estimating the parameter vector, $\beta$, the covariance matrix $\Delta$, which is the diagonal box matrix of the $\Delta_{i}$, is assumed to be known. Unfortunately, its heterogeneity parameter matrix, $\mathbf{T}$ has to be estimated from the data. The multivariate version of the DerSimonian and Laird methods of estimating the heterogeneity parameters are discussed and compared to the newly proposed methods in the following two subsections.

\subsection{The DerSimonian and Laird (DL) Extended Multivariate Estimators}

The extended multivariate meta-analysis of the DL method, as specified by Jackson et al. (2009), requires a pairwise estimation of the diagonal and off-diagonal elements of the heterogeneity matrix separately. Hence, the method handles any multivariate model as a collection of several interdependent bivariate models in the estimation procedures. It treats a p-dimensional matrix as a collection of $p_{\mathscr{C}_{2}}$ possible combinations of $2 \times 2$ matrices and builds its way up to the p-dimensional covariance matrix of $p(p+1) / 2$ elements. To estimate the $2 \times 2$ heterogeneity parameter matrix, the method considers a subset bivariate random-effects model with three elements of heterogeneity parameters. Hence, in the Jackson et al. (2009) method, there is no statistical methodology in place to estimate a $p \times p$ matrix of heterogeneity parameters beyond the $2 \times 2$ entries at a given time. This estimator, 


\section{A. Wouhib}

which referred to as DLJ, is criticized by Chen et al. (2012) for failing to produce a valid matrix estimate. Chen et al. (2012), however, also acknowledge that neither theirs nor Jackson's method would provide an estimator of the between-study covariance matrix that is always positive semi-definite, especially when heterogeneity is low.

In Jackson's method, the computation of an estimate for each pairwise entry (i.e., a separate $2 \times 2$ diagonal and off-diagonal elements) of the T matrix is accomplished by examining an appropriate bivariate model until exhausting all $\mathrm{p}$-dimensional variables to estimate a $p \times p$ matrix of heterogeneity parameters. However, the procedure in estimating the diagonal elements of the matrix is quite similar to the DL method of estimating a univariate heterogeneity parameter. Jackson et al. (2009) regards the DLJ method as an extended version of the univariate DL estimator in estimating $T_{D L J}$ from $2 \times 2$ Q-matrix.

The Q-matrix for all $u$ and $v$ such that, $1 \leq u<v \leq p$ is,

$$
Q_{u v}=\left(\begin{array}{cc}
\sum_{i=1}^{k} \frac{\left(b_{i u}-\bar{\beta}_{u}\right)^{2}}{\sigma_{i u u}} & \sum_{i=1}^{k} \frac{\left(b_{i u}-\bar{\beta}_{u u}\right)\left(b_{i v}-\bar{\beta}_{v v}\right)}{\sqrt{\sigma_{i u u} \sigma_{i v v}}} \\
\sum_{i=1}^{k} \frac{\left(b_{i u}-\bar{\beta}_{u u}\right)\left(b_{i v}-\bar{\beta}_{v v}\right)}{\sqrt{\sigma_{i u u} \sigma_{i v v}}} & \sum_{i=1}^{k} \frac{\left(b_{i v}-\bar{\beta}_{v}\right)^{2}}{\sigma_{i v v}}
\end{array}\right) .
$$

Where,

$$
\begin{gathered}
\bar{\beta}_{\mathrm{u}}=\frac{\sum_{\mathrm{i}=1}^{\mathrm{k}} \frac{\mathrm{b}_{\mathrm{iu}}}{\sigma_{\mathrm{iuu}}}}{\sum_{\mathrm{i}=1}^{\mathrm{k}} \frac{1}{\sigma_{\mathrm{iuu}}}} \\
\bar{\beta}_{\mathrm{uu}}=\frac{\sum_{\mathrm{i}=1}^{\mathrm{k}} \frac{\mathrm{b}_{\mathrm{iu}}}{\sqrt{\sigma_{\mathrm{iuu}} \sigma_{\mathrm{ivv}}}}}{\sum_{\mathrm{i}=1}^{\mathrm{k}} \frac{1}{\sqrt{\sigma_{\mathrm{iuu}} \sigma_{\mathrm{ivv}}}}} \\
\bar{\beta}_{\mathrm{v}}=\frac{\sum_{\mathrm{i}=1}^{\mathrm{k}} \frac{\mathrm{b}_{\mathrm{iv}}}{\sigma_{\mathrm{ivv}}}}{\sum_{\mathrm{i}=1}^{\mathrm{k}} \frac{1}{\sigma_{\mathrm{ivv}}}}
\end{gathered}
$$

and

$$
\bar{\beta}_{\mathrm{vv}}=\frac{\sum_{\mathrm{i}=1}^{\mathrm{k}} \frac{\mathrm{b}_{\mathrm{iv}}}{\sqrt{\sigma_{\mathrm{iuu}} \sigma_{\mathrm{ivv}}}}}{\sum_{\mathrm{i}=1}^{\mathrm{k}} \frac{1}{\sqrt{\sigma_{\mathrm{iuu}} \sigma_{\mathrm{ivv}}}}} .
$$


The expectation of $Q_{u v}$ is given by

$$
\begin{aligned}
& E\left(\sum_{i=1}^{k} \frac{\left(b_{i u}-\bar{\beta}_{u}\right)^{2}}{\sigma_{i u u}}\right)=(k-1)+\left(\sum_{i=1}^{k} \sigma_{i u u}^{-1}-\sum_{i=1}^{k} \sigma_{i u u}^{-2} / \sum_{i=1}^{k} \sigma_{i u u}^{-1}\right) \tau_{u u}, \\
& E\left(\sum_{i=1}^{k} \frac{\left(b_{i v}-\bar{\beta}_{v}\right)^{2}}{\sigma_{i v v}}\right)=(k-1)+\left(\sum_{i=1}^{k} \sigma_{i v v}^{-1}-\sum_{i=1}^{k} \sigma_{i v v}^{-2} / \sum_{i=1}^{k} \sigma_{i v v}^{-1}\right) \tau_{v v}
\end{aligned}
$$

and for $G_{i}=\left(\sigma_{i u u} \sigma_{i v v}\right)^{-1 / 2}$,

$$
E\left(\sum_{i=1}^{k}\left(b_{i u}-\bar{\beta}_{u u}\right)\left(b_{i v}-\bar{\beta}_{v v}\right) G_{i}\right)=\left(\sum_{i=1}^{k} \rho_{i u v}-\frac{\sum_{i=1}^{k} \rho_{i u v} G_{i}}{\sum_{i=1}^{k} G_{i}}\right)+\left(\sum_{i=1}^{k} G_{i}-\frac{\sum_{i=1}^{k} G_{i}^{2}}{\sum_{i=1}^{k} G_{i}}\right) \tau_{u v} .
$$

The estimators for heterogeneity parameters, $\hat{\tau}_{u u}, \hat{\tau}_{v v}$ and $\hat{\tau}_{u v}=\hat{\tau}_{v u}$ can be obtained by matching moments in equation (2.17), (2.18) and (2.19) respectively. Once a pairwise estimation procedure as described above is implemented, then the matrix, $\widehat{T}_{D L J}$, is formed as a collection of the pairwise estimates. Then the overall mean effect size, $\beta$, is estimated by

$$
\hat{\beta}_{D L J}=\left(\sum_{i=1}^{k} \widehat{W}_{i}\right)^{-1} \sum_{i=1}^{k} \widehat{W}_{i} \hat{\beta}_{i},
$$

where

$$
\widehat{W}_{i}=\left(\widehat{T}_{D L J}+\Sigma_{i}\right)
$$

The other DL extension method of estimating a multivariate heterogeneity parameter matrix is presented by Chen et al. (2012). Unlike the DLJ, this method, which is called DLC was initiated by extending the univariate DL estimation procedure in matrix form, and they estimated the heterogeneity parameters simultaneously. The estimation procedures are explained in the following steps: Let

$$
\Psi=\operatorname{Cov}\left(\hat{\beta}_{F}\right)=\left(Z^{\prime} \Sigma^{-1} Z\right)^{-1}=\left(\sum_{j=1}^{k} \Sigma_{j}^{-1}\right)^{-1}
$$

and

$$
\begin{gathered}
\Phi=\Psi^{-1}-\sum_{i=1}^{k} \Sigma_{i}^{-1} \Psi \Sigma_{i}^{-1} \\
A=\sum_{j=1}^{k} \Sigma_{j}^{-1}\left(b_{j}-\hat{\beta}_{F}\right)\left(b_{j}-\hat{\beta}_{F}\right)^{\prime}-(k-1) I_{p \times p},
\end{gathered}
$$

where

$$
Z=\left(I_{p \times p} \ldots I_{p \times p}\right)_{k p \times p}^{\prime},
$$


a stack of $p \times p$ identity matrices, and $\hat{\beta}_{\mathrm{F}}$ is an estimator for a fixed-effects model, and $(1 \leq j \leq k)$. Then, $\mathrm{E}(\mathrm{A})=\Phi \mathrm{T}$, for $T$ is the heterogeneity parameter matrix of size $p \times p$. Finally, to maintain its symmetric property, the DLC estimator matrix is re-written as:

$$
\widehat{T}_{D L C}=\frac{\Phi^{-1} A+A^{\prime} \Phi^{-1}}{2}
$$

The authors of the DLC method stressed that their method provides similar results as the Jackson's DLJ method and conducted simulations to support their claim.

In both extended multivariate DL methods, a remedy was proposed if the diagonals of $\widehat{T}_{D L J}$ and $\widehat{T}_{D L C}$ turned out to be negative. The remedy truncates the matrices to make them positive semidefinite. Although the newly made positive semi-definite matrices are acknowledged to be biased, it is difficult to draw similarities with truncating negative heterogeneity parameters in univariate models.

In their recent paper, Jackson, et al. (2013) endorsed the extended DerSimonian and Laird method of Chen et al. (2012) as a multivariate generalization of estimating the heterogeneity parameter matrix. Jackson et al. (2013) stated that their new method can handle missing data, can adjust for covariates in a meta-regression, and reduces to the method of Chen et al. (2012) with complete data and no covariates. Like the method by Chen et al. (2012), the new Jackson et al. (2013) method is based on matrix operations and invariant to linear transformations.

\subsection{The Sidik Jonkman(SJ) \& the Hybrid Methods of Estimating Heterogeneity Parameters}

The SJ multivariate method for estimating the heterogeneity parameter matrix emulates the SJ method of estimating the heterogeneity parameter under a univariate meta-analysis model. In the univariate meta-analysis, the fundamental difference of the SJ method from others is the assumption of $\tau^{2}>0$. Equivalently, the SJ method in a multivariate random-effects model is the assumption of heterogeneity parameter matrix, which is always positive definite and invertible.

From the random-effects model, the expectation for the $i^{\text {th }}$ study is

$$
E\left(\mathrm{~b}_{i}\right)=\beta,
$$

and the variance is

$$
\operatorname{var}\left(b_{i}\right)=\left(\mathrm{T}+\Sigma_{i}\right)
$$

If

$$
R_{i}=T^{-1 / 2} \Sigma_{i} T^{-1 / 2}
$$

is known and if $T$ is positive definite, then the total covariance matrix for study $i$ can be rewritten as,

$$
\Sigma_{i}+T=T^{1 / 2}\left(R_{i}+I_{p \times p}\right) T^{1 / 2}=T^{1 / 2} V_{i} T^{1 / 2}
$$

for

$$
V_{i}=\left(R_{i}+I_{p \times p}\right) .
$$


Then by changing the center of the distribution,

$$
\left(\mathrm{b}_{\mathrm{i}}-\beta\right) \sim \mathscr{N}\left(0, \mathrm{~T}^{1 / 2} \mathrm{~V}_{\mathrm{i}} \mathrm{T}^{1 / 2}\right) .
$$

Since $\mathrm{V}_{i}$ is a positive definite matrix, its inverse, $\mathrm{V}_{\mathrm{i}}^{-1}$ is also positive definite. Let

$$
Q_{i}=V_{i}^{-1 / 2}=\left(T^{-1 / 2} \Sigma_{i} T^{-1 / 2}+I_{p \times p}\right)^{-1 / 2}
$$

then, $Q_{i} Q_{i}=V_{i}^{-1}$ and equivalently, $\mathrm{Q}_{\mathrm{i}}^{-1} \mathrm{Q}_{\mathrm{i}}^{-1}=\mathrm{V}_{\mathrm{i}}$. Note that $V_{i}$ and $Q_{i}$ are symmetric matrices. By multiplying $\left(\mathrm{b}_{i}-\beta\right)$ with $Q_{i}$ then,

$$
Q_{i}\left(\mathrm{~b}_{i}-\beta\right) \sim \mathscr{N}(0, \mathrm{~T}), \forall i=1,2, \ldots, k .
$$

The variance of the distribution $Q_{i}\left(b_{i}-\beta\right)$, namely, $Q_{i}\left(\Sigma_{i}+T\right) Q_{i}$, can be expressed as

$$
\begin{gathered}
\mathrm{Q}_{i}\left(\Sigma_{i}+T\right) Q_{i}=Q_{i} T^{1 / 2} V_{i} T^{1 / 2} Q_{i} \\
\quad=Q_{i} T^{1 / 2} Q_{i}^{-1} Q_{i}^{-1} T^{1 / 2} Q_{i}
\end{gathered}
$$

Taking the first component of (2.35), which is $Q_{i} T^{1 / 2} Q_{i}^{-1}$, and multiplying both ends by an identity matrix

$$
I=T^{1 / 4} T^{-1 / 4}=T^{-1 / 4} T^{1 / 4}
$$

we get

$$
Q_{i} T^{1 / 2} Q_{i}^{-1}=T^{1 / 4} T^{-1 / 4} Q_{i} T^{1 / 4} T^{1 / 4} Q_{i}^{-1} T^{-1 / 4} T^{1 / 4} .
$$

Further, the component

$$
\begin{gathered}
T^{-1 / 4} Q_{i} T^{1 / 4}=T^{-1 / 4}\left(T^{-1 / 2} \Sigma_{i} T^{-1 / 2}+I\right)^{-1 / 2} T^{1 / 4} \\
=\left(T^{-1 / 2}\left(T^{-1 / 2} \Sigma_{i} T^{-1 / 2}+I\right) T^{1 / 2}\right)^{-1 / 2} \\
=\left(T^{-1} \Sigma_{i}+I\right)^{-1 / 2} .
\end{gathered}
$$

Similarly,

$$
\begin{gathered}
T^{1 / 4} Q_{i}^{-1} T^{-1 / 4}=T^{1 / 4}\left(T^{-1 / 2} \Sigma_{i} T^{-1 / 2}+I\right)^{1 / 2} T^{-1 / 4} \\
=\left(T^{-1 / 2}\left(T^{-1 / 2} \Sigma_{i} T^{-1 / 2}+I\right) T^{1 / 2}\right)^{1 / 2} \\
=\left(T^{-1} \Sigma_{i}+I\right)^{1 / 2}
\end{gathered}
$$

Then, from equation (2.36),

$$
Q_{i} T^{1 / 2} Q_{i}^{-1}=T^{1 / 4}\left(T^{-1} \Sigma_{i}+I\right)^{-1 / 2}\left(T^{-1} \Sigma_{i}+I\right)^{1 / 2} T^{1 / 4}
$$




\section{A. Wouhib}

is simplified as

$$
T^{1 / 4} I T^{1 / 4}=T^{1 / 2}
$$

We may also note that the second component of (2.35), $Q_{i}^{-1} T^{1 / 2} Q_{i}$, is a transpose of $Q_{i} T^{1 / 2} Q_{i}^{-1}$, the first component. As a result,

$$
Q_{i}^{-1} T^{1 / 2} Q_{i}=\left(T^{1 / 2}\right)^{\prime}
$$

Note that if

$$
\mathrm{Q}_{i} T^{1 / 2} Q_{i}^{-1}=T^{1 / 2} \Longrightarrow Q_{i} T^{1 / 2}=T^{1 / 2} Q_{i}
$$

this implies that $T^{1 / 2}$ and $Q_{i}$ are commutative matrices.

Finally, the variance of

$$
\mathrm{Q}_{i}\left(b_{i}-\beta\right)=Q_{i} T^{1 / 2} V_{i} T^{1 / 2} Q_{i}=T,
$$

and hence the distribution,

$$
\mathrm{Q}_{i}\left(b_{i}-\beta\right) \sim \mathscr{N}(0, T)
$$

Applying the fundamental theory of the normal distribution,

$$
\left(\mathrm{Q}_{i}\left(\mathrm{~b}_{i}-\beta\right)\right)\left(\mathrm{Q}_{i}\left(\mathrm{~b}_{i}-\beta\right)\right)^{\prime} \sim W_{p}(1, \mathrm{~T}),
$$

a $p$-dimensional Wishart distribution with 1 degree of freedom and parameter $T, \forall i=1,2, \ldots, k$. Then the sum of $\mathrm{p}$-dimensional $k$ independent studies distributed as,

$$
\sqrt{k} \sum_{i=1}^{k}\left(\mathrm{Q}_{i}\left(\mathrm{~b}_{i}-\beta\right)\right) \sim \mathscr{N}(0, \mathrm{~T})
$$

If

$$
S=k \sum_{i=1}^{k}\left(\mathrm{Q}_{i}\left(\mathrm{~b}_{i}-\beta\right)\right)\left(\mathrm{Q}_{i}\left(\mathrm{~b}_{i}-\beta\right)\right)^{\prime}
$$

then

$$
S \sim W_{p}(k, \mathbf{T})
$$

Under the random-effects model, the generalized least square (GLS) estimator of the population effect size, $\beta$ is $\hat{\beta}_{\mathrm{gls}}$, where $\hat{\beta}_{\mathrm{gls}}=\left(\mathrm{Z}^{\prime} \Delta^{-1} \mathrm{Z}\right)^{-1}\left(\mathrm{Z}^{\prime} \Delta^{-1} \mathrm{~b}\right)$ is the Best Linear Unbiased Estimator (BLUE). The matrix, $Z_{\mathrm{kp} \times \mathrm{p}}$, is a stack of $k$ identity matrices of size $p \times p$, the covariance matrix $\Delta$ is a $k p \times k p$ box diagonal matrix of $\left(\Sigma_{\mathrm{i}}+\mathrm{T}\right)$, each of size $p \times p$ of matrices of $k$ studies of $i=1,2, \ldots, k$, and the random vector, $\mathrm{b}$, is a $k p \times 1$ stacked with $\mathrm{b}_{1}, \mathrm{~b}_{2}, \ldots, \mathrm{b}_{\mathrm{k}}$, each a $p \times 1$ vector. 
Since

$$
\hat{\beta}_{\mathrm{gls}}=\left(\sum_{\mathrm{i}=1}^{\mathrm{k}}\left(\Sigma_{\mathrm{i}}+\mathrm{T}\right)^{-1}\right)^{-1} \sum_{\mathrm{i}=1}^{\mathrm{k}}\left(\Sigma_{\mathrm{i}}+\mathrm{T}\right)^{-1} \mathrm{~b}_{\mathrm{i}}
$$

and also

$$
\hat{\beta}_{\mathrm{gls}} \sim N\left(\beta,\left(\sum_{i=1}^{k}\left(\Sigma_{\mathrm{i}}+\mathrm{T}\right)^{-1}\right)^{-1}\right)
$$

if

$$
S^{*}=\sum_{\mathrm{i}=1}^{\mathrm{k}}\left(\mathrm{Q}_{\mathrm{i}}\left(\mathrm{b}_{\mathrm{i}}-\hat{\beta}\right)\right)\left(\mathrm{Q}_{\mathrm{i}}\left(\mathrm{b}_{\mathrm{i}}-\hat{\beta}\right)\right)^{\prime},
$$

then from the properties of multivariate normal distribution,

$$
\mathrm{S}^{*}=\sum_{\mathrm{i}=1}^{\mathrm{k}}\left(\mathrm{Q}_{\mathrm{i}}\left(\mathrm{b}_{\mathrm{i}}-\hat{\beta}\right)\right)\left(\mathrm{b}_{\mathrm{i}}-\hat{\beta}\right)^{\prime}\left(\mathrm{Q}_{\mathrm{i}}\right)^{\prime} \sim \mathrm{W}_{\mathrm{p}}(\mathrm{k}-1, \mathrm{~T}),
$$

a Wishart distribution with $k-1$ degrees of freedom for known $\mathrm{Q}_{i}$. Obviously, the expectation,

$$
E\left(\mathrm{~S}^{*} / \mathrm{T}\right)=k-1
$$

implies that,

$$
\widehat{\mathrm{T}}=\mathrm{S}^{*} /(\mathrm{k}-1)
$$

is an unbiased estimator of T. But, $\mathrm{S}^{*}$ is a function of $\widehat{\beta}$ and needs to be estimated first. Clearly, the estimator $\hat{\mathrm{T}}$ is unbiased if the parameter $\mathrm{Q}_{\mathrm{i}}$ is known and fixed for all values of $i=1,2, \ldots k$. Unfortunately, $\hat{T}$ is a function of $\mathrm{Q}_{i}$ which is not known and has to be estimated from the data. In other words, since the matrix $T$ is component of $\mathrm{Q}_{\mathrm{i}}$ for the study $i$, a reasonable initial estimate of $T$ is needed to estimate $Q_{i}$. In the case of univariate meta-analysis, Sidik and Jonkman (2005), suggested an the average of the sums of the squares of the effect sizes to be an initial value for the heterogeneity parameter.

In the multivariate case, let the initial matrix for estimating $T$ be

$$
\widehat{T}_{0}=\sum_{i=1}^{k}\left(b_{i}-\bar{b}\right)^{\prime}\left(b_{i}-\bar{b}\right) / k
$$

and

$$
\bar{b}=\sum_{i=1}^{k} b_{j} / k
$$

an arithmetic mean vector of the effect sizes. Then, as in the univariate meta-analysis, the initial matrix for $\mathrm{Q}_{\mathrm{i}}$ can be estimated as

$$
\widehat{\mathrm{Q}}_{\mathrm{i}}^{0}=\hat{\mathrm{T}}_{0}^{1 / 2}\left(\Sigma_{\mathrm{i}}+\hat{\mathrm{T}}_{0}\right)^{-1 / 2}=\left(\mathrm{T}_{0}^{-1 / 2} \Sigma_{\mathrm{i}} \mathrm{T}_{0}^{-1 / 2}+\mathrm{I}_{\mathrm{p} \times \mathrm{p}}\right)^{-1 / 2} .
$$


Using $\widehat{\mathrm{Q}}_{\mathrm{i}}^{0}$, the initial value for $\beta$ can be estimated as

$$
\hat{\beta}^{0}=\left(\sum_{\mathrm{i}=1}^{\mathrm{k}}\left(\Sigma_{\mathrm{i}}+\hat{\mathrm{T}}_{0}\right)^{-1}\right)^{-1} \sum_{\mathrm{i}=1}^{\mathrm{k}}\left(\Sigma_{\mathrm{i}}+\hat{\mathrm{T}}_{0}\right)^{-1} \mathrm{~b}_{\mathrm{i}}
$$

Then, the SJ estimator of multivariate heterogeneity parameter matrix is

$$
\hat{\mathrm{T}}_{\mathrm{SJ}}=\sum_{\mathrm{i}=1}^{\mathrm{k}}\left(\mathrm{Q}_{\mathrm{i}}^{0}\left(\mathrm{~b}_{\mathrm{i}}-\hat{\beta}^{0}\right)\left(\mathrm{b}_{\mathrm{i}}-\hat{\beta}^{0}\right)^{\prime} \mathrm{Q}_{\mathrm{i}}^{0^{\prime}}\right) /(\mathrm{k}-1) .
$$

Once the heterogeneity parameter matrix is estimated, the overall effect size vector, $\beta$ can be estimated as

$$
\widehat{\beta}_{\mathrm{SJ}}=\left(\sum_{\mathrm{i}=1}^{\mathrm{k}}\left(\Sigma_{\mathrm{i}}+\hat{\mathrm{T}}_{\mathrm{SJ}}\right)^{-1}\right)^{-1} \sum_{\mathrm{i}=1}^{\mathrm{k}}\left(\Sigma_{\mathrm{i}}+\hat{\mathrm{T}}_{\mathrm{SJ}}\right)^{-1} \mathrm{~b}_{\mathrm{i}}
$$

The SJ estimator is a general heterogeneity variance estimator that can be applied to any randomeffects model regardless of its dimension. In a univariate case, Sidik and Jonkman (2005) stated that the estimator does not share a shortcoming of other estimators because it always yields a nonnegative estimate. Moreover, it is derived from the weighted residual sum of squares in the framework of a linear regression model without covariates, where the weight is formulated in terms of the ratio of the study-specific variance to the heterogeneity variance. The SJ multivariate heterogeneity variance parameter matrix estimator is an extension of the univariate SJ method and should have the same properties as its univariate counterpart. Hence, under minimal heterogeneity, $T_{S J}$, has a tendency of overestimating the true matrix of heterogeneity parameters. As a consequence, exploring an alternative method in estimating the heterogeneity parameter matrix, which is based on the SJ method and that addresses the over-estimation concern, is important. The new estimator, which is called the Hybrid method, maintains all the desired properties of the SJ method and performs much better in the area where the SJ method reveals some concerns, especially when heterogeneity is minimal. Like SJ, the Hybrid method also assumes the heterogeneity true matrix to be positive definite matrix. This method is called the Hybrid method because the SJ estimator initiates an iterative procedure to estimate the heterogeneity parameters in a form of matrix. For the Hybrid method, first, a convergence criterion to stop the iteration has to be established and the SJ estimators, $\hat{T}_{S J}$ and $\hat{\beta}_{\mathrm{SJ}}$ are used as initial estimates in the first iteration. Then the second round of iteration takes place using $\hat{T}_{S J}$ and $\hat{\beta}_{\mathrm{SJ}}$ in equation (2.47) to estimate the second round heterogeneity parameter matrix estimator, $\hat{\mathrm{T}}_{2}$. Subsequently, the second round effect size vector, $\hat{\beta}_{2}$ is estimated by using $\hat{\mathrm{T}}_{2}$. The iteration procedure continues until the specified convergence criterion is met. If the criterion is met at the $j^{\text {th }}$ iteration, then $\hat{\mathrm{T}}_{\mathrm{j}}$ is designated as $\hat{\mathrm{T}}_{\mathrm{Hyb}}$, the Hybrid estimator of the heterogeneity parameter matrix. Similarly, the estimated overall effect size at the $j^{\text {th }}$ iteration, $\hat{\beta}_{\mathrm{j}}=\hat{\beta}_{\mathrm{Hyb}}$ is the Hybrid estimator for any dimensional multivariate random-effects meta-analysis.

\section{An illustrative Examples}

\subsection{Example I}

From the recent paper of Jackson, et al. (2013), a real study of hypertension treatment is taken as the first illustrative example. The example involves 10 studies that assess the effectiveness of hypertension treatment for lowering blood pressure. Each study provides complete data on two 
treatment effects, the difference in systolic blood pressure (SBP) and diastolic blood pressure (DBP) between the treatment and control groups, where these differences are adjusted for the participants' baseline blood pressures. A bigger reduction in blood pressure is a desirable outcome, so negative estimates indicate that the treatment is beneficial. The within-study correlations are known, so that the within-study covariance matrices are also known, and the data are shown in the following table below.

Table 1: Data on the Effectiveness of Hypertension Treatment for Lowering Blood Pressure

\begin{tabular}{cccccc}
\hline Study & $S B P$ & $S E_{S B P}$ & $D B P$ & $S E_{D B P}$ & $\rho$ \\
\hline 1 & -6.66 & 0.72 & -2.99 & 0.27 & 0.780 \\
2 & -14.17 & 4.73 & -7.87 & 1.44 & 0.450 \\
3 & -12.88 & 10.31 & -6.01 & 1.77 & 0.590 \\
4 & -8.71 & 0.30 & -5.11 & 0.10 & 0.770 \\
5 & -8.70 & 0.14 & -4.64 & 0.05 & 0.660 \\
6 & -10.60 & 0.58 & -5.56 & 0.18 & 0.490 \\
7 & -11.36 & 0.30 & -3.98 & 0.27 & 0.500 \\
8 & -17.93 & 5.82 & -6.54 & 1.31 & 0.611 \\
9 & -6.55 & 0.41 & -2.08 & 0.11 & 0.451 \\
10 & -10.26 & 0.20 & -3.49 & 0.04 & 0.511 \\
\hline
\end{tabular}

In the Table 1, SBP and DBP are the treatment effects on the systolic and diastolic blood pressures, respectively. The within-study standard error corresponding to each estimate, $S E_{S B P}$ and $S E_{D B P}$, along with the study-level correlations denoted by $\rho$ are also given.

Results from the multivariate meta-analysis using the Jackson, et al. (2009) method, the Chen, et al. (2012) method along with the SJ and the Hybrid methods are shown in Table 2. The betweenstudy correlation estimates are also presented in this table.

In the presence of substantial heterogeneity, Sidik and Jonkman (2007) showed that the DerSimonian and Laird method tends to underestimate the population heterogeneity parameter. In their univariate simulation study, they showed that the underestimation exacerbates as heterogeneity increases. Both DLJ and DLC methods seem to underestimate the heterogeneity parameter matrix in example I. As a result of the underestimation, the off-diagonal parameter, $T_{0,1}$, has much smaller estimates for both DLJ and DLC methods, implying small correlation between SBP and DBP for the treatment effect across studies. However, the underestimated heterogeneity parameters do not seem to have much influence on the estimated effect sizes.

\subsection{Example II}

Here the High School Longitudinal Study of 2009 (HSLS:09), cited by Chen et al. (2012) on a three dimensional multivariate meta-analysis of random-effects model is revisited to estimate heterogeneity parameter matrices of $3 \times 3$ dimension. HSLS:09 is a nationally representative, longitudinal study of more than 21,000 ninth graders in 944 schools who were be followed through their secondary and postsecondary years. 


\section{A. Wouhib}

Table 2: Estimates of Regression Coefficients with Associated Covariances and Heterogeneity Parameter Matrix Using the Example on the Effectiveness of Hypertension Treatment

\begin{tabular}{lcccc}
\hline Parameter & $D L J$ & $D L C$ & $S J$ & $H y b$ \\
\hline$\beta_{0}$ & -9.1738 & -9.1906 & -9.8473 & -9.5531 \\
$\beta_{1}$ & -4.3367 & -4.3188 & -4.5127 & -4.5625 \\
$\operatorname{Var}\left(\hat{\beta}_{0}\right)$ & 0.2952 & 0.3082 & 1.3035 & 0.5526 \\
$\operatorname{Var}\left(\hat{\beta}_{1}\right)$ & 0.1325 & 0.1365 & 0.3087 & 0.4828 \\
$\operatorname{Cov}\left(\hat{\beta}_{0}, \hat{\beta}_{1}\right)$ & 0.0147 & 0.0439 & 0.4661 & 0.4030 \\
$T_{0,0}$ & 1.9473 & 2.0431 & 5.8147 & 4.3731 \\
$T_{1,1}$ & 1.0293 & 1.0931 & 3.2023 & 4.3685 \\
$T_{0,1}$ & 0.0598 & 0.2822 & 3.6243 & 3.5897 \\
$\rho_{T}$ & 0.0422 & 0.1889 & 0.8399 & 0.8213 \\
\hline
\end{tabular}

In the within-study level analyses, the interest was to know whether sex, socioeconomic status and sex by socio-economic status interaction are predictive of the mathematics standardized theta score. Regression was performed for each of the eight race groups and the regression coefficients were estimated. Once the summary results were available, the second stage of the analysis was to estimate a vector of the overall effect sizes and heterogeneity parameter matrix. The estimated regression coefficients and associated covariance matrix for each of the eight race groups in Table 1 are taken from Chen et al. (2012). The value $b_{i 1}$ is the regression coefficient for sex, $b_{i 2}$ is the regression coefficient for socioeconomic status score and $b_{i 3}$ is the regression coefficient for sex by socio-economic status score interaction for race group $i$.

Table 3: Summary of Regression Results for Eight Race Groups and Three Regression Coefficients

\begin{tabular}{ccccccccccc}
\hline & \multicolumn{3}{c}{ Effect Size } & \multicolumn{3}{c}{ Variance } & \multicolumn{3}{c}{ Covariance } \\
\cline { 2 - 11 }$R_{i}$ & $b_{1 i}$ & $b_{2 i}$ & $b_{3 i}$ & $b_{1 i}$ & $b_{2 i}$ & $b_{3 i}$ & $\left(b_{1 i}, b_{2 i}\right)$ & $\left(b_{1 i}, b_{3 i}\right)$ & $\left(b_{2 i}, b_{3 i}\right)$ \\
\hline 1 & 0.3161 & 7.4015 & 0.4278 & 2.3568 & 9.7029 & 4.4114 & -1.2105 & 0.8524 & -6.1753 \\
2 & -0.3201 & 6.9426 & -0.9816 & 0.2529 & 0.7016 & 0.2743 & 0.1498 & -0.1019 & -0.4167 \\
3 & 0.6983 & 4.6680 & -0.2415 & 0.1444 & 0.6481 & 0.2608 & -0.0652 & 0.0433 & -0.3899 \\
4 & 3.2736 & 4.3080 & 0.2052 & 3.8428 & 10.3517 & 4.8268 & -4.5587 & 3.2892 & -6.6684 \\
5 & -0.1599 & 5.6398 & -0.6782 & 0.1161 & 0.4363 & 0.1733 & -0.0992 & 0.0645 & -0.2610 \\
6 & -0.6989 & 6.3158 & -0.7918 & 0.1603 & 0.7697 & 0.3180 & 0.0242 & -0.0129 & -0.4686 \\
7 & -3.6094 & 9.3429 & -2.8711 & 3.2054 & 17.8889 & 7.2101 & -1.1984 & 0.8437 & -10.7697 \\
8 & 0.2172 & 6.4078 & -0.6093 & 0.0278 & 0.1184 & 0.0482 & 0.0136 & -0.0091 & -0.0716 \\
\hline
\end{tabular}


By applying the extended DL multivariate methods for estimating the between-study covariance matrix as described by Jackson et al. (2009), one can obtain the estimated matrix as,

$$
\hat{T}_{D L J}=\left(\begin{array}{ccc}
0.2079 & -0.2135 & 0.1179 \\
-0.2135 & -\mathbf{0 . 0 4 7 3} & 0.2616 \\
0.1179 & 0.2616 & -\mathbf{0 . 1 9 5 4}
\end{array}\right) .
$$

However, the estimated matrix has negative diagonal elements. As remedy to overcome this difficulties, Jackson et al. (2009) and Chen et al. (2012) proposed either to leave the estimated matrix as is or transform the original estimate to a positive semi-definite matrix by means of spectral decomposition. Since $\hat{T}_{D L J}$ is a symmetric matrix, it can be stated as, $\hat{T}_{D L J}=U D U^{\prime}$, where $U$ is a diagonal matrix with eigenvalues of $\hat{T}_{D L J}$. By replacing negative elements of $D$ with zeroes and renaming the diagonal matrix with replaced zeros as $D_{T}$, the transformed estimator would be $\hat{T}_{D L J T}=U D_{T} U^{\prime}$. But, the transformed positive semi-definite matrix is biased.

After applying the remedy to make the original negative definite matrix estimated by Jackson, et al. (2009), the transformed new positive semi-definite matrix is referred here as $\hat{T}_{D L J T}$, where

$$
\hat{T}_{D L J T}=\left(\begin{array}{ccc}
0.2557 & -0.1220 & 0.0097 \\
-0.1220 & 0.1279 & 0.0542 \\
0.0097 & 0.0542 & 0.0501
\end{array}\right) .
$$

Obviously, the new matrix is positive semi-definite but very different from the originally estimated matrix. However, both Jackson et al. (2009) and Chen et al. (2012) did not interpret the elements of the newly formed positive semi-definite matrix with respect to further statistical inferences, such as analyzing correlation between-studies. The estimates for the overall effect sizes and the associated covariance matrix using the corrected (transformed) heterogeneity parameter matrix is

$$
\begin{gathered}
\hat{\beta}_{D L J T}=\left(\begin{array}{c}
-0.0612 \\
6.1873 \\
-0.7038
\end{array}\right) \\
\widehat{\operatorname{Cov}}\left(\hat{\beta}_{D L J T}\right)=\left(\begin{array}{ccc}
0.0675 & -0.0241 & 0.0046 \\
-0.0241 & 0.0884 & -0.0302 \\
0.0046 & -0.0302 & 0.0357
\end{array}\right) .
\end{gathered}
$$

If the Chen's extended DL heterogeneity parameter matrix estimates in the original form is $\hat{T}_{D L C}$, then the estimated matrix before applying the remedy is,

$$
\hat{T}_{D L C}=\left(\begin{array}{ccc}
0.2553 & -0.1665 & 0.0857 \\
-0.1665 & -\mathbf{0 . 1 0 2 2} & 0.2958 \\
0.0857 & 0.2958 & -\mathbf{0 . 2 1 7 8}
\end{array}\right) .
$$

Similar to the DLJ, the estimator by Chen, et al. (2012), $\hat{T}_{D L C}$ also has negative diagonal elements and need to be a positive semi-definite matrix applying the proposed remedy. As the original 


\section{A. Wouhib}

DCL, the estimated matrix is modified by using the remedy to get rid of the negative diagonal elements in the matrix. The newly transformed matrix is called $\hat{T}_{D L C T}$ and it is

$$
\hat{T}_{D L C T}=\left(\begin{array}{ccc}
0.2805 & -0.0947 & 0.0031 \\
-0.0947 & 0.1025 & 0.0602 \\
0.0031 & 0.0602 & 0.0533
\end{array}\right)
$$

Similarly, the overall effect sizes and its associated covariance matrix based on the corrected heterogeneity covariance matrix of Chen et al. (2012) are estimated as $\hat{\beta}_{D L J T}$ and $\hat{C} O v_{\hat{\beta}_{D L T}}$ and displayed below:

$$
\begin{gathered}
\hat{\beta}_{D L C T}=\left(\begin{array}{c}
-0.0604 \\
6.1821 \\
0.7009
\end{array}\right) \\
\widehat{\operatorname{Cov}}\left(\hat{\beta}_{D L C T}\right)=\left(\begin{array}{ccc}
0.0720 & -0.0187 & 0.0030 \\
-0.0187 & 0.0834 & -0.0287 \\
0.0030 & -0.0287 & 0.0359
\end{array}\right)
\end{gathered}
$$

Using the SJ method for multivariate random-effects model, the heterogeneity parameter matrix, $\hat{\mathrm{T}}_{\mathrm{SJ}}$ is estimated as

$$
\hat{\mathbf{T}}_{\text {SJ }}=\left(\begin{array}{ccc}
3.5975 & -2.5579 & 1.7711 \\
-2.5579 & 2.3114 & -1.3155 \\
1.7711 & -1.3155 & 1.0233
\end{array}\right) .
$$

The SJ estimates for overall effect size and associated covariance matrix are

$$
\hat{\beta}_{\mathbf{S J}}=\left(\begin{array}{c}
-0.0604 \\
6.1855 \\
-0.6541
\end{array}\right)
$$

and

$$
\widehat{\operatorname{Cov}}\left(\hat{\beta}_{\mathbf{S J}}\right)=\left(\begin{array}{ccc}
0.0150 & 0.0056 & 0.0028 \\
0.0056 & 0.0968 & -0.0027 \\
0.0028 & -0.0027 & 0.0644
\end{array}\right)
$$

respectively.

Furthermore, when the Hybrid method is applied, convergence was slow and the iteration was longer than usual. That could be due to the ill-conditioned nature of the true heterogeneity parameter matrix or due to a very small heterogeneity in the data. Perhaps, it could be also the main reason for the estimated singular matrices in both the Jackson et al. (2009) and Chen et al. (2012) methods. The instability of the true heterogeneity parameter matrix slowed the iteration for convergence and as a consequence, the Hybrid method attained the maximum number of iterations set by a stopping rule. The stopping rule for estimating the heterogeneity parameter matrix is set to be, either a very small difference between matrices of successive iterations or a reasonable number of maximum 
iterations, whichever comes first. Because of this rear scenario, the estimated Hybrid heterogeneity parameter matrix is not as well conditioned and has a positive determinant that is close to zero.

$$
\hat{\mathbf{T}}_{\mathbf{H y b}}=\left(\begin{array}{ccc}
1.8070 & -1.7276 & -0.2946 \\
-1.7276 & 1.7373 & 0.3337 \\
-0.2946 & 0.3337 & 0.0798
\end{array}\right)
$$

Similarly, the estimated effect sizes and associated covariance matrix of the effect size are

$$
\hat{\beta}_{\mathbf{H y b}}=\left(\begin{array}{c}
-0.1527 \\
6.2307 \\
-0.6650
\end{array}\right)
$$

and

$$
\widehat{\operatorname{Cov}}\left(\hat{\beta}_{\mathbf{H y b}}\right)=\left(\begin{array}{ccc}
0.2726 & -0.2397 & -0.0360 \\
-0.2397 & 0.3057 & 0.0059 \\
-0.0360 & 0.0059 & 0.0384
\end{array}\right)
$$

Both $\hat{\mathrm{T}}_{\mathrm{SJ}}$ and $\hat{\mathrm{T}}_{\text {Hyb }}$ produce matrices with quadratic forms and they are positive definite. Despite the similarities in estimated effect sizes, the estimated heterogeneity parameter matrices are quite different from method to method in the above example. Although each method expected to produce different results, the outcome in this example exacerbated by the true heterogeneity parameter matrix of the data. The results are consistent with univariate cases as close to zero heterogeneity parameter slows the convergence process of the Hybrid method and produces negative estimates for other methods including the DerSimonian and Laird method (Wouhib, 2013).

\section{Simulation Studies}

After estimating the heterogeneity parameters in the example, a simulation study was performed by choosing appropriate population parameters. The simulation is based on three dimensional multivariate data populated with study numbers, $k=10$ with unequal sample sizes, $n_{i}=12,13, \ldots, 17$ for $i=1,2, \ldots, 10$ and 1,000 replications. In the simulation, $\mathrm{T}$, a $3 \times 3$ matrix of heterogeneity parameters and $\beta$, a three-dimensional zero vector, were used as true population covariance and mean respectively. The within-study covariance matrices were populated similar to Jackson et al. (2009) as stated in section 5.1 for 3-dimensional analysis. The vector for the population effect size is set to be zero since it has no major impact on the heterogeneity parameter matrix estimation procedures. The between-study heterogeneity parameter matrix and the effect size vector of the population in the simulation are

$$
\mathbf{T}=\left(\begin{array}{ccc}
1.8070 & -1.7276 & -0.2946 \\
-1.7276 & 1.7373 & 0.3337 \\
-0.2946 & 0.3337 & 0.0798
\end{array}\right)
$$

and

$$
\beta=\left(\begin{array}{l}
0.00 \\
0.00 \\
0.00
\end{array}\right)
$$




\section{A. Wouhib}

respectively.

Using the Jackson et al. (2009) method for extended DL, the estimated heterogeneity parameter matrix and the vector of overall effect sizes are computed from the simulation as $\hat{T}_{D L J}$ and $\hat{\beta}_{D L J}$ respectively and presented as

$$
\hat{\mathbf{T}}_{\text {DLJ }}=\left(\begin{array}{ccc}
1.82359 & -1.70926 & -0.29111 \\
-1.70926 & 1.75092 & 0.32293 \\
-0.29111 & 0.32293 & 0.09129
\end{array}\right) .
$$

The effect size vector along with the corresponding covariance matrix are also estimated as

$$
\hat{\beta}_{\mathrm{DLJ}}=\left(\begin{array}{c}
-0.00749 \\
-0.01433 \\
0.00278
\end{array}\right)
$$

and

$$
\widehat{\operatorname{Cov}}\left(\hat{\beta}_{\mathbf{D L J}}\right)=\left(\begin{array}{ccc}
0.18457 & -0.16941 & -0.02838 \\
-0.16941 & 0.17735 & 0.03246 \\
-0.02838 & 0.03246 & 0.01272
\end{array}\right)
$$

Based on the Chen et al. (2012) method, the estimated heterogeneity parameter matrix is

$$
\hat{T}_{D L C}=\left(\begin{array}{ccc}
1.93840 & -1.61902 & -0.30542 \\
-1.61902 & 1.91788 & 0.35403 \\
-0.30542 & 0.35403 & 0.17044
\end{array}\right),
$$

and similar to the DLJ, the effect size vector for DLC is also estimated as

$$
\hat{\beta}_{\text {DLC }}=\left(\begin{array}{c}
-0.00566 \\
0.00523 \\
-0.04659
\end{array}\right) \text {, }
$$

along with the corresponding covariance matrix,

$$
\widehat{\operatorname{Cov}}\left(\hat{\beta}_{\mathbf{D L C}}\right)=\left(\begin{array}{ccc}
0.19665 & -0.15986 & -0.02928 \\
-0.15986 & 0.19458 & 0.03598 \\
-0.02928 & 0.03598 & 0.02075
\end{array}\right) .
$$

The estimated matrix using the SJ method for heterogeneity parameters, the estimated vector for effect sizes, and associated covariance matrix are stated as follows:

$$
\begin{gathered}
\hat{\mathbf{T}}_{\mathbf{S J}}=\left(\begin{array}{ccc}
1.84236 & -1.72513 & -0.29268 \\
-1.72513 & 1.76380 & 0.33049 \\
-0.29268 & 0.33049 & 0.11337
\end{array}\right), \\
\hat{\beta}_{\mathbf{S J}}=\left(\begin{array}{c}
0.00148 \\
-0.00527 \\
0.00234
\end{array}\right),
\end{gathered}
$$


and

$$
\widehat{\operatorname{Cov}}\left(\hat{\beta}_{\mathbf{S J}}\right)=\left(\begin{array}{ccc}
0.17098 & -0.15140 & -0.02424 \\
-0.15140 & 0.16400 & 0.03014 \\
-0.02424 & 0.03014 & 0.018487
\end{array}\right) .
$$

The Hybrid method estimates of heterogeneity matrix and the effect sizes vector are presented along with the associated covariance matrix of the estimated effect sizes as

$$
\begin{gathered}
\hat{\mathbf{T}}_{\mathbf{H y b}}=\left(\begin{array}{ccc}
1.83654 & -1.73297 & -0.29747 \\
-1.73297 & 1.75359 & 0.33286 \\
-0.29747 & 0.33286 & 0.09842
\end{array}\right), \\
\hat{\beta}_{\mathbf{H y b}}=\left(\begin{array}{c}
0.00238 \\
-0.00411 \\
0.00183
\end{array}\right),
\end{gathered}
$$

and

$$
\widehat{\operatorname{Cov}}\left(\hat{\beta}_{\mathbf{H y b}}\right)=\left(\begin{array}{ccc}
0.18605 & -0.17169 & -0.02876 \\
-0.17169 & 0.17765 & 0.03363 \\
-0.028761 & 0.03363 & 0.01350
\end{array}\right)
$$

respectively.

In this simulation, the heterogeneity parameter matrix, which was taken from the Hybrid method estimate of Example II is not stable with a determinant very close to zero. As explained earlier, this is mainly due the nature of the data used in the example or the condition of the true heterogeneity parameter matrix itself. Because of that, the simulation produced (not shown) negative definite matrices in more than $74 \%$ and $67 \%$ cases of the DLJ and DLC methods respectively, when estimating the true heterogeneity parameter matrix. Hence, the negative definite matrices from these methods were transformed to non-negative definite form using the remedy proposed by Jackson, et al. (2009) and Chen, et al. (2012). Obviously, the estimates are biased and the remedy in correcting the matrices may affect estimates of the population heterogeneity parameter matrix for these methods and consequently, the estimated effect sizes are also affected. Perhaps, the DLJ and DLC methods are unable to work properly where the true population heterogeneity parameter matrix is close to homogeneity as seen in this simulation. Nevertheless, in multiple other sets of simulations [24], where the population heterogeneity parameter matrix was selected to be stable and substantial, the DLJ and the DLC methods estimated the heterogeneity parameters with a very small proportion of negative definite matrices with the tendency of overestimation (Example I). In the case of substantial heterogeneity, still the SJ and the Hybrid methods have shown much better results in estimating both the population heterogeneity parameter matrix as shown in the first example. We should also notice that both the SJ and the Hybrid methods do not need correction remedies and the estimates for the heterogeneity parameter matrix are always positive definite. 
A. Wouhib

\section{Discussion and Conclusion}

From the simulation, both the SJ and the Hybrid estimators of the of the heterogeneity parameter matrix performed better than the Chen et al. (2012) method in terms of proximity to the true heterogeneity matrix and in producing always non-negative definite matrices. Moreover, the simulation showed that the Hybrid estimator does better than the SJ estimator for both diagonal and off-diagonal elements of the heterogeneity parameter matrix. Although our simulation does not include Maximum Likelihood (ML) and Restricted Maximum Likelihood (REML) methods, in a close to homogeneity cases, these methods are likely to produce estimates with negative definite matrix, similar to the DL methods. Truncating a negative definite matrix by means of the spectral decomposition not only changes the negative definite matrix into positive semi-definite form of nonzero diagonal element, but it also changes the off-diagonal elements of the matrix. This can lead to a meaningless interpretation of the elements of the truncated matrix. Moreover, unlike the widely used truncation in the univariate meta-analysis, the application of spectral decomposition may has unintended consequences in multivariate meta-analysis when transforming a negative definite heterogeneity matrix to a nonnegative form. As we have seen in the second example, estimating the between-study correlation coefficients could be misleading when estimated heterogeneity parameter matrices of DLJ and DLC are used after transformation.

The SJ and the Hybrid methods have important features in estimating matrices of heterogeneity parameters. The estimated matrices are always positive definite, and no need for matrix truncation or spectral decomposition. Similar to a one-way random-effects meta-analysis, the newly proposed methods are viable alternatives and perhaps the only option when other methods fail to properly estimate a matrix of heterogeneity parameters for a multivariate random-effects model(Wouhib, 2013). The SJ and the Hybrid methods perform nicely with any multidimensional meta-analysis whether heterogeneity is substantial or minimal. Among these two methods, the simulation showed that the Hybrid method does much better in estimating the true heterogeneity parameter matrix in terms of closeness to the true parameters of the between-study covariance matrix. Although the Hybrid method is iterative; Wouhib (2013) has shown that it usually converges faster than other iterative methods including ML and REML.

\section{Acknowledgments}

Special thanks go to Dr. Bimal Sinha for his valuable time in reviewing and guiding this work. Also, I would like to thank my colleagues at NCHS for their support.

\section{References}

[1] T. W. Anderson, An Introduction to Multivariate Statistical Analysis, 3rd ed. (Wiley: New York), (2003).

[2] W. L. Baker, et al. Understanding heterogeneity in meta-analysis: the role of meta-regression, The International Journal of Clinical Practice, 63 (2009) 1426-1434.

[3] C. S. Berkey, et al. Meta-Analysis of Multiple Outcomes by Regression with Random-Effects, Statistics in Medicine, 17, (1998) 2537-2550.

[4] B. J. Betsy and M. J. Wu, The Synthesis of Regression Slopes in Meta-Analysis, Statistical Science, 22, (2007), 414-429.

[5] H. Chen, A. K. Manning and J. Dupuis, A Method of Moments Estimator for Random Effect Multivariate meta-analysis, Biometrics, 68, (2012), 12781284

[6] R. DerSimonian and N. Laird, Meta-analysis in clinical trials, Controlled Clinical Trials, 7, (1986), 177-188. 
[7] J. Hartung, G. Knapp and B. K. Sinha, Statistical meta-analysis with applications,(Wiley), (2008).

[8] D. A. Harville, Matrix Algebra from a Statistician's Prospective, (Springer), (1997).

[9] H. Cv. Houwelingen, L. R. Arends and T. Stijnen, Advanced methods in meta-analysis: multivariate approach and meta-regression, Statistics in Medicine, 21, (2002), 589 - 624.

[10] D. Jackson, I. R. White and R. Riley, A Matrix-based method of moments for fitting the multivariate random effects model for meta-analysis and meta-regression, Biometrical Journal, 55, (2013), 231 245.

[11] D. Jackson, R.D. Riley and I. R. White, Multivariate meta-analysis: Potential and promise, Statistics in Medicine 30, (2011), 2481 - 2498.

[12] D. Jackson, I.R. White and S. G. Thompson, Extending DerSimonian and Laird's methodology to perform multivariate random-effects meta-analysis, Statistics in Medicine, 29, (2010), 1282-1297 .

[13] D. Jackson, I.R. White and R. D. Riley, A matrix-based method of moments for fitting the multivariate random effects model for meta-analysis and meta-regression, Biometrical Journal 55, (2013), 231245

[14] G. Knapp and J. Hartung, Improved tests for a random effects meta-regression with a single covariate, Statistics in Medicine, 22, (2003), 2693-2710.

[15] D. Mavridis and G. Salanti, A Practical Introduction to Multivariate Meta-Analysis, Statistical Methods in Medical Research, January 23, 2012.

[16] S. W. Raudenbush, Random Effects Models. In H. C. Cooper and L. V. Hedges (Eds.), The Handbook of Research Synthesis. (New York: Russell Sage Foundation) (1994), 301-321.

[17] R. D. Riley, Multivariate meta-analysis: the effect of ignoring within-study correlation, Journal of the Royal Statistical Society: Series A, 172, (2009), 789-811.

[18] R. D. Riley, et al., Bivariate random effects meta-analysis and the estimation of between-study correlation, BMC Medical Research Methodology, 7 (2007).

[19] R. D. Riley, J. R. Thompson and K. R. Abrams, An alternative model for bivariate random-effects meta-analysis when the within-study correlations are unknown, Biostatistics, 9, (2008), 172-186.

[20] J. Sánchez-Meca and F. Marín-Martínez, Confidence Intervals for the Overall Effect Size in RandomEffects Meta-Analysis, Psychological Methods, 13, (2008), 3148.

[21] K. Sidik and J. N. Jonkman, Simple heterogeneity variance estimation for meta-analysis, Journal of Royal Statistical Society Series C, 54, (2005), 367-384.

[22] K. Sidik and J. N. Jonkman, A Note on Variance Estimation in Random Effects Meta-Regression, Journal of Biopharmaceutical Statistics, 15, (2006), 823-838.

[23] K. Sidik and J. N. Jonkman, A comparison of heterogeneity variance estimators in combining results of studies, Statistics in Medicine, 26, (2007), 1964 - 1981.

[24] A. Wouhib, Exploring an Alternative Method in Estimating Heterogeneity Parameters in RandomEffects Meta-Analysis, Doctoral Thesis, (Department of Mathematics and Statistics, UMBC), (2013). 